\title{
Effects of Water Activity on Growth and Sporulation of Paecilomyces farinosus in Liquid and Solid Media
}

\author{
By JEANNE M. M. INCH AND A. P. J. TRINCI* \\ Microbiology Group, Department of Cell and Structural Biology, School of Biological Sciences, \\ Williamson Building, University of Manchester, Manchester M13 9PL, UK
}

(Received 15 August 1986)

\begin{abstract}
The effects of water activity on colony radial growth rate, specific growth rate, growth yield and blastospore production of Paecilomyces farinosus were studied. The compound (PEG 200) used to adjust water activity was not used for growth either when it was the sole carbon source in the medium or when the medium also contained glucose. Daily additions of water were made to cultures ('compensated' cultures) to compensate for evaporation; when this was not done the water activity of the medium decreased during incubation and a water activity at which growth ceased was eventually attained. A correlation was observed between the inhibitory effects of water activity on specific growth rate $(\mu)$ in 'compensated' shake flask cultures and on colony radial growth rate $\left(K_{\mathrm{r}}\right)$ on solid medium. Thus, $K_{\mathrm{r}}$ can be used to assess the effects of water activity on mould growth. Growth yield decreased linearly with decrease in water activity, and a decrease in water activity from 0.985 to 0.958 caused an approximately sixfold increase in the yield of blastospores per unit biomass dry weight.
\end{abstract}

\section{INTRODUCTION}

Trinci (1971) showed that the radial growth rate of a fungal colony $\left(K_{\mathrm{r}}\right)$ is a function of the width of its peripheral growth zone $(w)$ and the organism's specific growth rate $(\mu)$. Thus,

$$
K_{\mathrm{r}}=w \mu
$$

It follows from equation (1) that colony radial growth rate can only be used as a reliable indicator of specific growth rate when the width of the colony's peripheral growth zone is not altered by the experimental variable being considered. For example, temperature does not have an appreciable effect on peripheral growth zone width, and therefore colony radial growth rate can be used to determine the optimum temperature for mould growth (Trinci, 1971). By contrast, although L-sorbose has no appreciable effect on the maximum specific growth rate of Neurospora crassa, it causes a considerable decrease in peripheral growth zone width and hence in colony radial growth rate on solid medium (Trinci \& Collinge, 1973). Similarly, although the colony radial growth rate of Aspergillus nidulans varies with glucose concentration over the range 0.01 to $80 \mathrm{~g}$ glucose $1^{-1}$ (Trinci, 1969), glucose concentration would only be expected to influence the mould's maximum specific growth at the lowest extreme of this range (Monod, 1942; Fiddy \& Trinci, 1975). Thus, colony radial growth does not always provide a reliable way of assessing the effect of an environmental variable on mould growth.

Although colony radial growth rate is often used to determine the effect of water activity on fungal growth (Luard \& Griffin, 1981; Luard, 1985; Kuthubutheen \& Webster, 1986; Magan \& Lynch, 1986; Eamus \& Jennings, 1986), no study has been made to assess the validity of this practice. In this paper we show that when the growth rate of Paecilomyces farinosus is altered by changing the water activity of the medium, there is a direct relationship between specific growth rate in liquid media and colony radial growth rate on solid medium. 


\section{METHODS}

Organism and media. Paecilomyces farinosus (Holm ex S. F. Gray) Brown \& Smith was obtained from the Glasshouse Crops Research Institute, Littlehampton, UK. The defined medium (DM) used has been described previously (Trinci, 1971); it was buffered at $\mathrm{pH} 6.8$ with $0.05 \mathrm{M}$ (final concentration) phosphate buffer and was prepared and sterilized as described by Trinci (1971). When necessary, medium was solidified with agar (final concentration $15 \mathrm{~g}^{-1}$; Taiyo, Davis Gelatine). Polyethylene glycol of $M_{\mathrm{r}} 200$ (PEG 200) was obtained from Sigma and was sterilized $\left(121^{\circ} \mathrm{C}\right.$ for $\left.15 \mathrm{~min}\right)$ in water (liquid medium) or water agar (solid medium); the final concentration of PEG 200 in media ranged from 0.5 to $1.25 \mathrm{M}$, giving water activities $\left(a_{\mathrm{w}}\right)$ of $0.985-0.947$ for liquid media and 0.994-0.946 for solid media.

Measurement of water activity. The water potentials of liquid and solid media were determined using an HR 33T dew point microvoltmeter (Wescor) and water activities were calculated from these measurements (Griffin, 1981).

Inoculation, cultural conditions and growth measurements. Shake flask cultures were prepared by inoculating $20 \mathrm{ml}$ volumes of media in $250 \mathrm{ml}$ nephlos flasks (Trinci, 1972) with $1 \mathrm{ml}$ volumes of a blastospore suspension. The blastospore inoculum was obtained by filtering stationary phase $(65 \mathrm{~h})$ cultures of $P$. farinosus through two layers of sterile lens tissue (Whatman). All shake flask cultures were incubated at $25^{\circ} \mathrm{C}$ and 200 r.p.m. on a rotary shaker with a $2.5 \mathrm{~cm}$ stroke; incubations lasted up to $600 \mathrm{~h}$ (initial $a_{\mathrm{w}} 0.947$ ). Under these experimental conditions each flask lost about $0.5 \mathrm{ml}$ of water per day by evaporation. Therefore, in some experiments (lasting $350 \mathrm{~h}$ ) daily additions of sterile distilled water (at $25^{\circ} \mathrm{C}$ ) were made to compensate for evaporation.

Petri dishes $(9 \mathrm{~cm}$ diameter) containing $20 \mathrm{ml}$ volumes of solid media were put in perspex boxes $(28 \times 16 \times 10 \mathrm{~cm})$ and left to equilibrate at $25^{\circ} \mathrm{C}$ for $72 \mathrm{~h}$. Five plates in each box were then inoculated with plugs ( $5 \mathrm{~mm}$ diameter) taken from a spread plate inoculated with $1.0 \mathrm{ml}$ of a conidial suspension, and incubated at $25^{\circ} \mathrm{C}$ for $24 \mathrm{~h}$; the remaining five plates in each box were left uninoculated and helped to maintain the relative humidity in the box at an appropriate level.

Colony diameter was measured using a Shadowmaster (Baty \& Co., Burgess Hill, Sussex, UK) with a $\times 10$ objective. Culture optical density was measured using an EEL colorimeter (Evans Electroselenium) with a green filter $(540-560 \mathrm{~nm})$. Each measurement was the mean of three replicate flasks. Dry weights were determined after filtering cultures through previously washed, dried and weighed Whatman no. 5 filter papers in Buchner flasks. The biomass on the filter was washed with $100 \mathrm{ml}$ distilled water and then dried at $70{ }^{\circ} \mathrm{C}$ to constant weight. Counts of blastospores were made in early stationary phase using an improved Neubauer haemocytometer. Each result was the mean of nine replicates and the standard error was determined. Analysis of variance $(95 \%$ confidence level) was calculated after transformation of the data to $\log _{10}$.

Glucose determinations. Glucose was measured using the Reflocheck system (Boehringer Mannheim).

\section{RESULTS AND DISCUSSION}

$P$. farinosus had a specific growth rate of $0 \cdot 116 \mathrm{~h}^{-1}$ on DM $\left(a_{\mathrm{w}} 0.985\right)$ and a slightly lower specific growth rate of $0.110 \mathrm{~h}^{-1}$ on DM containing $0.5 \mathrm{M}$-PEG $200\left(a_{\mathrm{w}} 0.977\right)$. This result suggests that PEG 200 is inhibitory to growth because of its effect on water activity rather than because it is toxic. Fig. 1 shows growth of 'compensated' (daily additions of water to replace that lost by evaporation) and 'non-compensated' batch cultures of $P$. farinosus. Cessation of growth of 'compensated' cultures (initial $a_{\mathrm{w}} 0.949$ ) was associated with glucose exhaustion. By contrast the deceleration in the rate of growth of the 'non-compensated' cultures (initial $a_{\mathrm{w}} 0.954$ ) which occurred after $120 \mathrm{~h}$ incubation was associated with a decrease in water activity to about 0.940 . After $325 \mathrm{~h}$ incubation, the 'non-compensated' cultures had stopped growing although the medium still contained $18.4 \mathrm{~mm}$ glucose; presumably evaporation had resulted in the establishment of a water activity $(0.92)$ which was inhibitory to further growth. This method (allowing evaporation to increase water activity) could be used to determine the water activity at which mould growth ceases.

Figs 2 and 3 and Table 1 show the effects of water activity on growth and sporulation of 'compensated' shake flask cultures of $P$. farinosus. At water activities of 0.977 (DM with 0.5 MPEG 200) and below, there was a reduction in specific growth rate with decrease in water activity of the medium; over the range of water activities from 0.947 to 0.977 there was a direct relationship between the logarithm of specific growth rate and the water activity of the medium (Fig. 3). The basis of this relationship is not known. Watson (1970) also found that the specific growth rate of batch cultures of Saccharomyces cerevisiae decreased with decrease in water activity of the medium.

PEG 200 was not used for growth of $P$. farinosus when it was the sole carbon source supplied in 


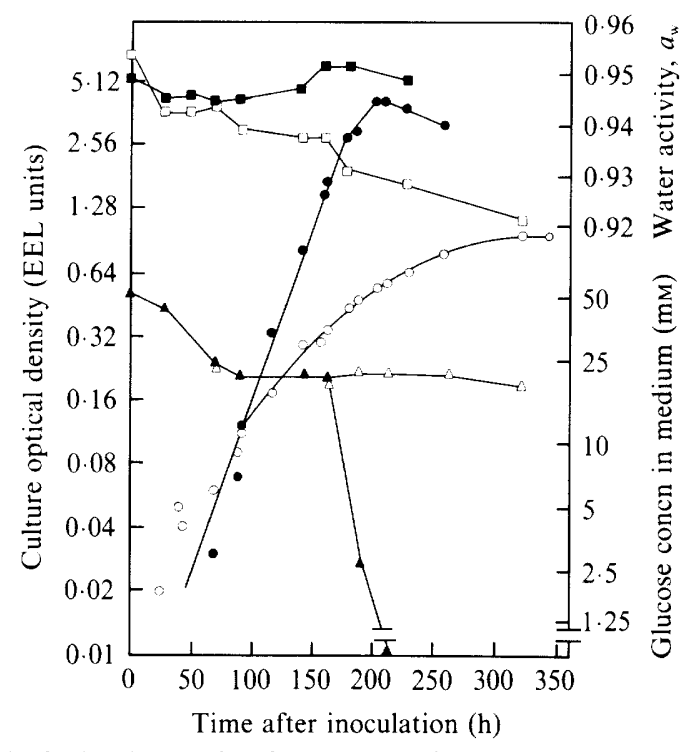

Fig. 1. Growth of shake flask cultures of $P$. farinosus at $25^{\circ} \mathrm{C}$ in DM containing $1 \cdot 1 \mathrm{M}-\mathrm{PEG} 200$. Daily additions of water were made to some cultures $(0)$ to maintain the water activity approximately constant; no additions were made to other cultures $(O)$. The water activities of 'compensated' (water added) ( $\square$ ) and 'non-compensated' $(\square)$ cultures, and the glucose concentrations of 'compensated' $(\boldsymbol{\Delta})$ and 'non-compensated' $(\triangle)$ cultures were also measured.

Table 1. Effects of water activity on specific growth, colony radial growth rate, growth yield and sporulation of $P$. farinosus at $25^{\circ} \mathrm{C}$ on DM containing various concentrations of PEG 200

Sterile distilled water was added to the shake flask cultures at daily intervals to compensate for evaporation.

Growth of 'compensated' shake flask cultures

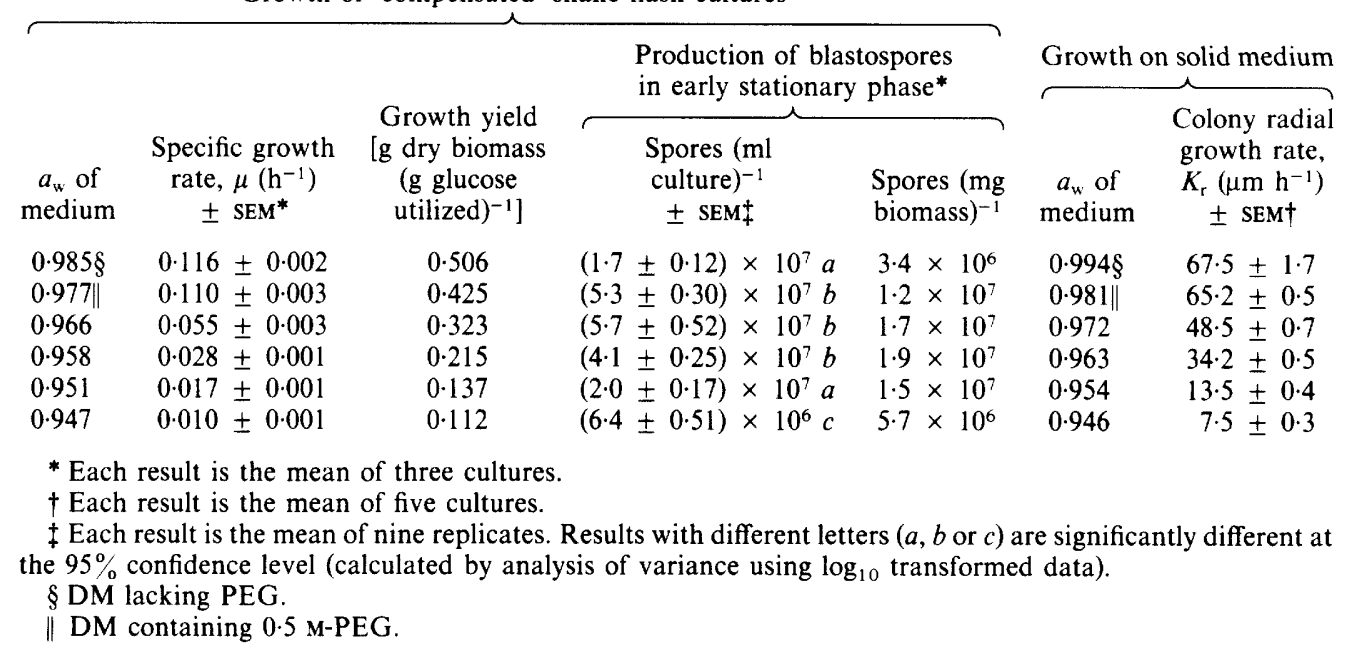

the medium (result not shown), and $0.5 \mathrm{M}-\mathrm{PEG}$ (final concentration in the medium) did not have an appreciable effect on specific growth rate or cause an increase in the final biomass of cultures grown on glucose (Table 1). However, bacteria which can utilize PEG 200 as a carbon source have been isolated and characterized (Kawai et al., 1984).

The growth yield [g biomass produced (g glucose utilized) $)^{-1}$ ] of $P$. farinosus was reduced from 


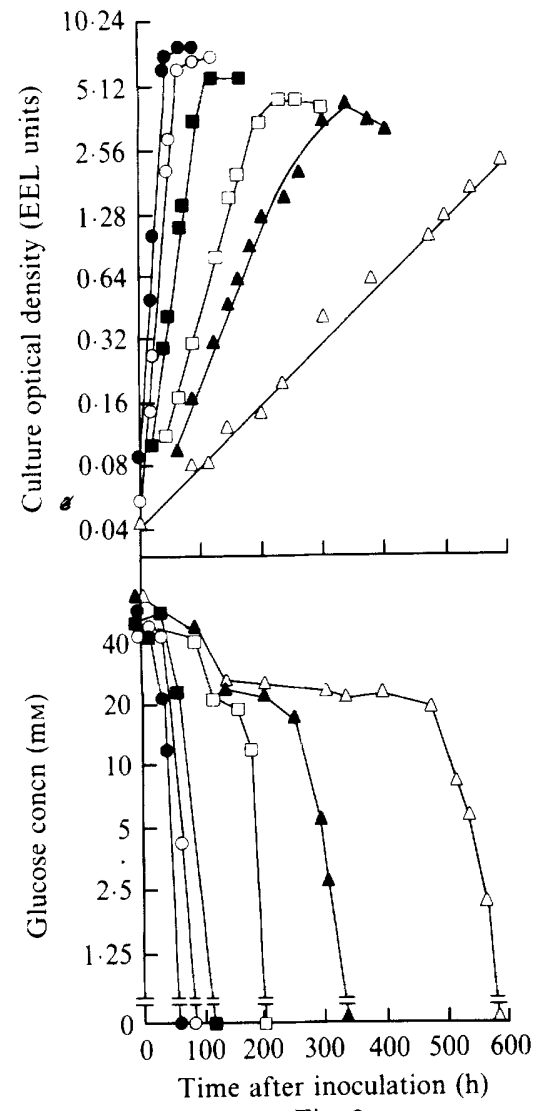

Fig. 2

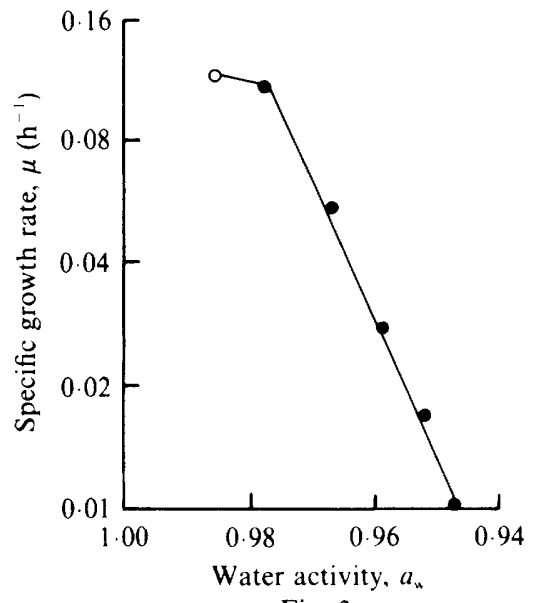

Fig. 3

Fig. 2. Growth and glucose concentrations of 'compensated' (daily additions of water) shake flask cultures of $P$. farinosus at $25^{\circ} \mathrm{C}$ in DM (O) and in DM containing various concentrations of PEG 200; the water activities of the media were $0.985(\bigcirc), 0.977(\bigcirc), 0.966(\square), 0.958(\square), 0.957(\Delta)$ and 0.947 $(\triangle)$.

Fig. 3. Effect of water activity on the specific growth rate of 'compensated' (daily additions of water) shake flask cultures of $P$. farinosus at $25^{\circ} \mathrm{C}$ on DM (O) and DM containing various concentrations of PEG 200 (O).

0.506 at $a_{\mathrm{w}} 0.985(\mathrm{DM})$ to 0.112 at $a_{\mathrm{w}} 0.947$ (DM with 1.25 M-PEG 200) (Table 1) and a linear relationship was observed between decrease in the water activity of the medium and decrease in growth yield (Fig. 4). This relationship reflects the increased requirement of $P$. farinosus for maintenance energy (Pirt, 1965) at low water activities and this can be largely attributed to the increased requirement of the organism for energy for 'osmotic work' and the reduction in its specific growth rate. Watson (1970) also found that the growth yield of $S$. cerevisiae in batch culture was reduced when the water activity of the medium was lowered by addition of $\mathrm{NaCl}$.

The effects of water activity on the specific growth rate of $P$. farinosus in submerged culture and on colony radial growth rate on solid medium are compared in Fig. 5. The results suggest that specific growth rate and colony radial growth rate are affected by water activity to a similar extent and that therefore colony radial growth rate is a valid way of assessing the effect of water activity on mould growth. The result also suggests that, over the PEG 200 range tested, water activity has no appreciable effect on colony peripheral growth zone width (equation 1).

Finally, Table 1 shows the effect of water activity on the number of blastospores produced by $P$. farinosus in submerged culture; at low water activity there was a significant increase in 


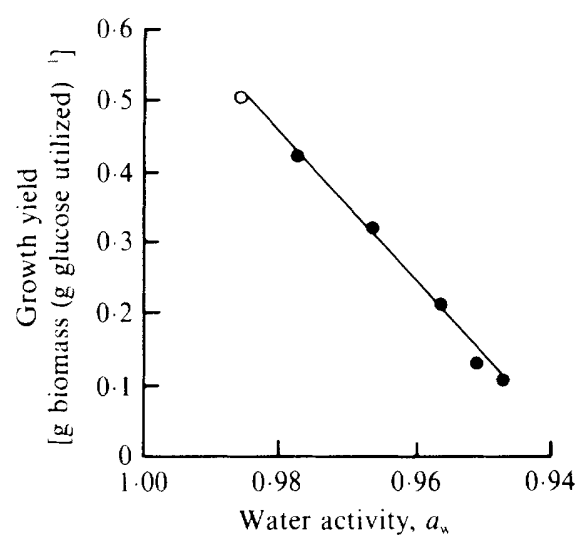

Fig. 4

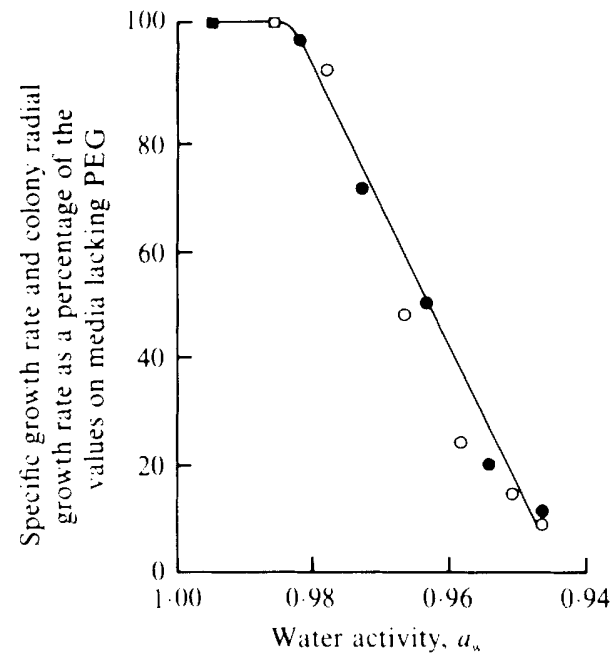

Fig. 5

Fig. 4. Effect of water activity on the growth rate of 'compensated' (daily additions of water) shake flask cultures of $P$. farinosus at $25^{\circ} \mathrm{C}$ on DM $(O)$ and DM containing various concentrations of PEG 200 (O).

Fig. 5. Comparison of the effects of water activity on the specific growth rate $(\square, \bigcirc)$ and colony radial growth rate $(\square, 0)$ of cultures of $P$. farinosus at $25^{\circ} \mathrm{C}$ on DM $(\square, \square)$ and DM containing various concentrations of PEG $200(\mathrm{O}, \mathrm{O})$. Growth rates are expressed as percentages of the growth rate obtained on DM alone.

number of blastospores formed per unit biomass. $P$. farinosus is a pathogen of brown planthopper (Nilaparvata lugens) and the present results suggest that blastospore yield of this fungus in submerged culture may be increased by adjusting the water activity of the culture medium.

We thank Dr M. J. Earnshaw for help with the dew point microvoltimeter, Dr A. T. Gillespie of the Glasshouse Crops Research Institute for providing the $P$. farinosus isolate and the EEC for a grant to support this research.

\section{REFERENCES}

EAmus, D. \& Jennings, D. H. (1986). Turgor and fungal growth: studies on water relations of mycelia of Serpula lacrimans and Phallus impudicus. Transactions of the British Mycological Society 86, 527-535.

Fiddy, C. \& Trincl, A. P. J. (1975). Kinetics and morphology of glucose-limited cultures of moulds grown in a chemostat and on solid media. Archives of Microbiology 103, 191-197.

Griffin, D. H. (1981). In Fungal Physiology, p. 153. New York: Wiley.

LUARD, E. J. (1985). Respiration of Penicillium chrysogenum in relation to the osmotic potential of the growth medium. Experimental Mycology 9, 99-107.

LUARD, E. J. \& GRIFFIN, D. M. (1981). Effect of water potential on fungal growth and turgor. Transactions of the British Mycological Society 76, 33-40.

Kawai, F., Kimura, T., Tani, Y. \& Yamada, H. (1984). Involvement of a polyethylene glycol (PEG)oxidizing enzyme in the bacterial metabolism of PEG. Journal of Agricultural and Biological Chemistry 48, 1349-1351.

KuthubutheEN, A. J. \& Webster, J. (1986). Effects of water availability on germination, growth and sporulation of coprophilous fungi. Transactions of the British Mycological Society 86, 77-91.

MAGAN, N. \& LYNCH, J. M. (1986). Water potential, growth and cellulolysis of fungi involved in decomposition of cereal residues. Journal of General Microbiology 132, 1181-1187.

Monod, J. (1942). Recherches sur la Croissance des Cultures Bactériennes. Paris: Hermann.

PIRT, S. J. (1965). The maintenance energy of bacteria in growing cultures. Proceedings of the Royal Society B163, 224-231.

TrincI, A. P. J. (1969). A kinetic study of the growth of Aspergillus nidulans and other fungi. Journal of General Microbiology 57, 11-24.

Trinci, A. P. J. (1971). Influence of the peripheral growth zone on the radial growth rate of fungal colonies. Journal of General Microbiology 67, 325334.

TrincI, A. P. J. (1972). Culture turbidity as a parameter of mould growth. Transactions of the British Mycological Society 58, 467-473. 
Trinci, A. P. J. \& Collinge, A. J. (1973). Influence of L-sorbose on the growth and morphology of Neurospora crassa. Journal of General Microbiology 78, 179192.
Watson, T. G. (1970). Effects of sodium chloride on steady-state growth and metabolism of Saccharomyces cerevisiae. Journal of General Microbiology 64, 91-99. 